\title{
Further evidence that psychopathology networks have limited replicability and utility: Response to Borsboom et al. and Steinley et al
}

\author{
Miriam K. Forbes, \\ University of Minnesota \\ Aidan G. C. Wright, \\ University of Pittsburgh \\ Kristian E. Markon, and \\ University of lowa \\ Robert F. Krueger \\ University of Minnesota
}

\section{Abstract}

In our target article, we tested the replicability of four popular psychopathology network estimation methods that aim to reveal causal relationships among symptoms of mental illness. We started with the focal data set from the two foundational psychopathology network papers (i.e., the National Comorbidity Survey-Replication) and identified the National Survey of Mental Health and Wellbeing as a close methodological match for comparison. We compared the psychopathology networks estimated in each dataset—as well as in ten sets of random split-halves within each dataset-with the goal of quantifying the replicability of the network parameters as they are interpreted in the extant psychopathology network literature. We concluded that current psychopathology network methods have limited replicability both within and between samples, and thus have limited utility. Here we respond to the two commentaries on our target article, concluding that Steinley, Hoffman, Brusco and Sher's (2017) findings-along with other recent developments in the literature-provide further conclusive evidence that psychopathology networks have poor replicability and utility.

\section{Introduction}

The two commentaries on our target article (Forbes, Wright, Markon, \& Krueger, 2017), from advocates of psychopathology networks (Borsboom et al., 2017) and scholars versed in multivariate statistical methodology (Steinley, Hoffman, Brusco \& Sher, 2017) differ substantially in their assessment of our findings. Borsboom et al. question the accuracy and validity of our work, and present a re-analysis of the data that they suggest provides

Please note that the published version of the target article (Forbes, Wright, Markon, \& Krueger, this issue) includes corrections made to the results for the directed acyclic graphs in the preprint that was available on the Open Science Framework from April 7th-24th 2017. 
evidence that "network models replicate very well" (p. 3). We argue this conclusion is not consistent with the data, and is at odds with emerging evidence in the literature as well as with many of their own previously stated conclusions. Steinley et al. describe an innovative method to test whether network parameters and measures of correspondence between networks differ from what would be expected in random data (i.e., by chance). They conclude that "the problem is likely worse" than our results indicated (p. 1). Our conclusions closely align with Steinley et al., and we consider their proposed method to be a key contribution to strengthening the network literature. Given space constraints here, we therefore spend much of this response briefly addressing the key issues raised by Borsboom et al. (see online supplement Table S1 for our response to some of the less central points). We then focus on evidence from others' work-including Steinley et al.'s commentary-that the replicability of psychopathology networks remains a substantial problem. Indeed, replicability is only one of many problems facing this new field.

\section{Borsboom et al.'s main criticisms of our article}

The use of zero-imputation to account for the skip-structure in the structured diagnostic interviews of the two surveys biased our results-It is remarkable that Borsboom et al. chastise us for this, given they used the same process on the same data to argue for the utility of network models (Borbsoom \& Cramer, 2013; Cramer et al., 2010). In fact, this was their methodological decision, which we emulated in an effort to mirror their methods- "We interpreted missing values that arose from the skip structure of the questionnaire as absent symptoms and replaced these by zeros, which seems a reasonable course of action given the way the DSM-IV is set up." (Borsboom \& Cramer, 2013, p. 104). We subsequently emulated this approach in the NSMHWB data, as have all other network analyses of psychopathology data based on structured interviews with a skip-structure that we know of (e.g., Boschloo et al., 2015; Rhemtulla et al., 2016). Zero-imputation is thus a potential limitation of extant network approaches, rather than a "statistical inaccuracy" (p. 15) of our work.

\section{The methods we used to estimate the relative importance networks render} these results invalid-Our goal was to test the replicability of the psychopathology networks as they are interpreted in the extant literature. Studies that include relative importance networks routinely emphasise stronger edges with high relative importance in their interpretation - particularly with respect to purported clinical utility (e.g., Bryant et al., 2017; Heeren \& McNally, 2016; Hoorelbeke, Marchetti, De Schryver \& Koster, 2016; McNally, 2016; Robinaugh, LeBlanc, Vuletich \& McNally, 2014). We therefore chose to examine the replicability of these edges, as well as of uncensored relative importance networks (i.e., with all edges estimated). However, Borsboom et al. point out that our censoring rule for the censored relative importance networks had the unintended effect of removing both edges in pairs with nearly identical edge weights. This point is well taken and we are happy to accept that these particular results may not reflect the expected replicability of the extant relative importance network literature. We also note that in Borsboom et al.'s analyses the relative importance networks had better replicability—and may therefore represent a better avenue for future research — than the putatively "state-of-the-art" Ising models, which are used in the majority of psychopathology network research. 
The assumptions underlying our methods were misguided-Borsboom et al. stress "the importance of assessing stability and replicability of network structures stands beyond doubt" (p. 5). They also emphasise their openness to evidence that network models are flawed in these regards, as this would represent progress towards researchers' common goal of robust and replicable scientific knowledge. ${ }^{1}$ However, they readily reject the results of our target article, suggesting that the design of our study was not appropriate for assessing replicability. Specifically, they suggest that it is not reasonable to expect that networks should replicate in methodologically matched samples_-or even in two random halves of a single dataset (p. 25-26). This position is perplexing to us, given that generalisability and replicability are both fundamental to the utility of research (e.g., why estimate psychopathology networks at all if we do not think they are able to identify meaningful relationships?). In fact, a review paper from their own group specifically recommends "cross-validation across similar samples" to investigate replicability (Fried \& Cramer, in press, p. 38).

Borsboom et al. also argue that networks should not be expected to replicate across different network estimation methods (e.g., Ising models, relative importance networks, and directed acyclic graphs [DAGs]), citing the fact that they each "get at different aspects of the data" (p. $22)^{2}$ and differ in sensitivity and specificity. However, Borsboom et al. do propose that concordance between methods can be examined based on a nesting approach that tests only the edges estimated in sparser networks (e.g., DAGs) against the edges estimated in less sparse networks (e.g., Ising models). Their suggested approach highlights the predictable conclusion that the edges from the sparser DAGs were replicated in the less sparse Ising models within each sample, but offers no guidance for how to interpret the other crossmethod replicability results in their online materials (i.e., that only $41.3-42.5 \%$ of the edges in the Ising models were replicated in the DAGs within and between samples; and that all DAG and Ising model pairs had different nodes with the highest in strength, out strength, and closeness centrality). Their conclusion that "Cross-method replication could hardly be better." certainly appears to be unwarranted (p. 12).

In short, Borsboom et al. suggest that the replicability of network models cannot be tested based on the comparisons of different samples or different methods. Despite this, based on these same comparisons they conclude that networks models replicate very well across different samples and different methods (when using the "right" metrics for quantifying replicability).

We used the wrong metrics for quantifying replicability-Borbsoom et al. state "the main problem with Forbes et al.'s assessment of replicability is that they do not use any measures that would seem of immediate relevance to any such analysis" (p. 18). It is hard to determine how they arrived at this conclusion ${ }^{3}$, given our metrics were specifically selected

\footnotetext{
${ }_{1}^{1}$ Indeed, this goal was the ultimate motivation for writing our target article.

${ }^{2}$ This line of argument highlights how important it is that researchers stop using these methods interchangeably as if they identify the same inferential targets (i.e., dynamic causal relationships among symptoms). Instead, it is vital to take the time to determine which method, if any, can uncover true underlying models (e.g., in simulated data with a known structure). Similarly, Borsboom et al. suggest that different centrality measures (e.g., strength, closeness, betweenness) should not be used either interchangeably or in conjunction with one another to identify the most central or influential node in a network. Importantly, both points contradict current practices as reflected in published studies, and represent important new messages for researchers using psychopathology network methods.
} 
to quantify replicability of the network parameters at the level they are used and interpreted in the literature. For example, the key results from our target article are based on the research questions "is edge A-B estimated in both networks?", if so "how much did the strength of this edge change from network 1 to 2?", and "does node A have the highest (or second, third, fourth highest, etc.) centrality in both networks?". These characteristics represent the features of psychopathology networks that correspond to network theory, and their purported clinical utility (Borsboom, 2017; Fried et al., 2016). In contrast, "the most intuitive and important" of the metrics Borsboom et al. propose for assessing replicability (p. 7) is a Pearson correlation between the edge lists of a pair of networks. This translates to the research question "at a global level, how similar is the list of all edges estimated in network 1 to the list of all edges estimated in network 2". This metric does not have any clear relevance to the inferences psychopathology networks are promoted to provide.

Borsboom et al.'s proposed methods for quantifying replicability "led to results directly opposed" to ours (p. 3) and this is most clear when comparing the conclusions that our study and the two commentaries arrived at regarding the Ising models in the full samples (see Table 1). The differences are remarkable and highlight that not only do Borsboom et al.'s proposed methods fail to align with the level that network parameters are interpreted, but that they have a striking lack of sensitivity. This lack of sensitivity is highly problematic, as it instils a false sense of confidence in results that evidently lack validity. Even when holding all the characteristics of the networks equal between samples (i.e., identical node selection, similar sample size, the same study methods, and the same analytic method) the key details of the model do not replicate. Combined with the results of Steinley et al., it is clear to us that although the global characteristics appear to be replicable (e.g., the number of edges and average edge strength ${ }^{4}$ ), the detailed parameters of psychopathology networks as currently formulated are too empirically weak to provide a meaningful basis for understanding psychopathology, much less building meaningful theories.

\section{Evidence of poor replicability from the psychopathology network literature}

Borsboom et al. claim that "inadequacy of the data and analyses" (p. 25) in our target article invalidates the results. ${ }^{5}$ Not surprisingly, we disagree. However, even if this claim were true, one need only look at the emerging network literature to find an abundance of evidence for problems in replicating the results of network analyses. Take, for example, the eight papers we are aware of that examine PTSD symptom networks (i.e., not including other disorders or covariates) in adult samples using putatively "state-of-the-art" network methods: Armour, Fried, Deserno, Tsai and Pietrzak, 2017; Birkeland and Heir, 2017; Epskamp, Borsboom and Fried, 2017; Fried et al., under review; McNally, Heeren and Robinaugh, 2017; Mitchell et al., 2017; Spiller et al., 2017; and Sullivan, Smith, Lewis and Jones, 2016. All eight papers

\footnotetext{
${ }^{3}$ As well as how they determined our indices of replicability to be "debatable" (p. 6), while labelling their own as "intuitive" (p. 7) and "powerful tools" (p. 8).

${ }^{4}$ We also note that interpreting the global network characteristics requires caution; for example, Terluin et al. (2016) found that the differences in edge strength between symptoms can be artefactual, representing differential range restriction across severity subgroups (i.e., should not be interpreted as differences in impact of one symptom on another).

${ }^{5}$ We remind the reader that these were the same data and analyses used in the major foundational papers for these methods (Borsboom \& Cramer, 2013; Cramer et al., 2010) and the basis for Borsboom et al.'s (this issue) conclusion that "network models replicate very well” (p. 3).
} 
use graphical LASSO regularisation to eliminate weak and unreliable edges in conjunction with the bootnet package (Epskamp et al., 2017) to "safeguard against false positive results, and also help us to identify consistent pathways that are highly reliable across studies" (Fried \& Cramer, in press, p. 40). Between these eight papers, fourteen (87.5\%) of the sixteen symptoms common to DSM-IV and DSM-5 PTSD diagnostic criteria were reported in-text as having particularly high centrality (see Table 2). Six (42.9\%) of these purportedly highly influential symptoms were reported as highly central in only a single paper, and none $(0 \%)$ in a majority of the papers. ${ }^{6}$ Similarly, there were fourteen specific edges emphasised as strong and reliable between the eight papers (see Table 3 ). Only one (7.1\%) of these edges was consistently estimated as a strong relationship (i.e., hypervigilance-startle); others varied substantially in strength, and seven (50\%) were absent altogether from the PTSD network in at least one of the papers.

In short, the PTSD network literature shows that neither gLASSO regularisation nor bootnet are sufficient for identifying reasonably replicable results. This finding highlights the central role that Steinley et al.'s proposed method should have in psychopathology network research going forward. As Steinley et al. illustrate, network parameters that are significant and stable but not different from random chance are uninteresting and unlikely to replicate. ${ }^{7}$ It also leaves us in the difficult situation of not knowing what, if anything, can be meaningfully concluded from the extant network literature with respect to the onset, maintenance, or treatment of psychopathology.

\section{The future of psychopathology networks}

The road ahead for empirical psychopathology network research is challenging. While network theory (e.g., Borsboom, 2017) calls attention to interesting and potentially important avenues for future research, the current psychopathology network methodologies are plagued with substantial flaws. For example, in the few months since our target article was accepted for publication, a series of papers have emerged on substantial problems facing the methods, in addition to poor replicability (Bos et al., 2017; Epskamp et al., 2017; Fried \& Cramer, in press; Guloksuz, Pries \& van Os, 2017; Steinley et al., 2017; Wichers, Wigman, Bringmann \& de Jonge, 2017). Taken together, this growing literature highlights the remarkable sensitivity of psychopathology network results to a multitude of factors including the study design, variables included, characteristics of the data, and analytic methods (e.g., Bulteel, Tuerlinckx, Brose \& Ceulemans, 2016; Terluin, de Boer \& de Vet, 2016; Wichers et al., 2017). The generalizability of psychopathology networks is further compromised by the fact that cross-sectional symptom networks change over time (Bryant et al., 2017), do not reflect temporal symptom dynamics derived in the same data (i.e., have different symptom-to-symptom associations and different central symptoms; Bos et al., 2017), and that group-level networks are not expected to generalize to individuals (Borsboom \& Cramer, 2013; Fried \& Cramer, in press; Fried et al., 2016).

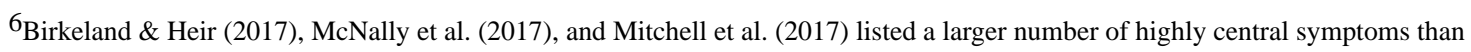
was supported by bootnet and made this distinction in-text. If we narrow it down to counting only their "bootnet-central" symptoms, eleven symptoms were reported as highly central across the eight papers; five $(45.5 \%)$ in only a single paper, and none $(0 \%)$ in the majority of papers.

${ }^{7}$ All but one (97.8\%) of the estimated edges identified by Steinley et al. as significantly different from chance in the NCS-R Ising model was replicated in the NSMWHB Ising model.
} 
It therefore seems fair to conclude — as we did in our target article- that cross-sectional psychopathology networks evidently have limited utility. We reiterate the warning of Steinley et al. that continuing to apply the current methods "runs the very real risk of creating a series of publications that contain results that are not reproducible and likely no different than what is expected under one of the most basic models of chance..." (p. 23). Developing appropriate methods to ensure the reliability and validity of the results is thus a crucial step that needs to be addressed before the proliferation of psychopathology networks continues.

Ultimately, there is a fundamental disconnect between network theory-which has a central tenet that "mental disorders arise from the causal interaction between symptoms in a network" (Borsboom, 2017, p. 6) - and the vast majority of contemporary network research. Current cross-sectional network study designs and methods do not and cannot test the research questions or hypotheses of network theory (Guloksuz et al., 2017; Wichers et al., 2017). Fried and Cramer (in press) thus conclude that it is up to network researchers "to be careful not to over-interpret results of network analyses as representing reality" (p. 20), and cross-sectional psychopathology networks are instead often framed as a hypothesisgenerating exercise (e.g., Borsboom \& Cramer, 2013; Fried \& Cramer, in press). However, each hypothesis regarding the clinical importance of an individual symptom, or the existence of dynamic causal relationships between symptoms (e.g., each result in Tables 2 and 3) requires an extensive program of research based on the collection of comprehensive longitudinal and/or experimental data. In a few short years, a multitude of hypotheses have been generated in psychopathology network research. It is now time to start the hard work of testing these hypotheses using appropriate study designs and analytic methods.

\section{Supplementary Material}

Refer to Web version on PubMed Central for supplementary material.

\section{Acknowledgments}

Miriam Forbes' work on this commentary response was supported by a NIDA training grant (T320A037183). Aidan Wright's efforts were supported by NIMH (L30MH101760). Robert Krueger's efforts were supported by NIH (R01AG053217; U19AG051426) and by the Templeton Foundation.

\section{References}

Armour C, Fried E, Deserno M, Tsai J, Pietrzak R. A network analysis of DSM-5 posttraumatic stress disorder symptoms and correlates in US military veterans. Journal of Anxiety Disorders. 2017; 45:49-59. [PubMed: 27936411]

Birkeland M, Heir T. Making connections: Exploring the centrality of posttraumatic stress symptoms and covariates after a terrorist attack. European Journal of Psychotraumatology. 2017; 8:1333387. [PubMed: 29038689]

Borsboom D. A network theory of mental disorders. World Psychiatry. 2017; 16:5-13. DOI: 10.1002/ wps.20375 [PubMed: 28127906]

Borsboom D, Cramer A. Network analysis: An integrative approach to the structure of psychopathology. Annu Rev Clin Psychol. 2013; 9:91-121. DOI: 10.1146/annurevclinpsy-050212-185608 [PubMed: 23537483] 
Borsboom D, Fried E, Epskamp S, Waldorp L, van Borkulo C, van der Maas H, Cramer A. False alarm? A comprehensive reanalysis of "Evidence that psychopathology symptom networks have limited replicability” by Forbes, Wright, Markon, and Krueger. J Abnorm Psychol. 2017

Bos F, Snippe E, de Vos S, Hartmann J, Simons C, van der Krieke L, ... Wichers M. Can we jump from cross-sectional to dynamic interpretations of networks? Implications for the network perspective in psychiatry. Psychother Psychosom. 2017; 86:175-177. [PubMed: 28490028]

Boschloo L, van Borkulo C, Rhemtulla M, Keyes K, Borsboom D, Schoevers R. The network structure of symptoms of the Diagnostic and Statistical Manual of Mental Disorders. PLoS One. 2015; 10:e0137621.doi: 10.1371/journal.pone.0137621 [PubMed: 26368008]

Bryant R, Creamer M, O’Donnell M, Forbes D, McFarlane A, Silove D, Hadzi-Pavlovic D. Acute and chronic posttraumatic stress symptoms in the emergence of posttraumatic stress disorder: A network analysis. JAMA Psychiatry. 2017; 74:135-142. DOI: 10.1001/jamapsychiatry.2016.3470 [PubMed: 28002832]

Bulteel K, Tuerlinckx F, Brose A, Ceulemans E. Using raw VAR regression coefficients to build networks can be misleading. Multivariate behavioral research. 2016; 51:330-344. [PubMed: 27028486]

Cramer A, Waldorp L, van der Maas H, Borsboom D. Comorbidity: A network perspective. Behavioral and Brain Sciences. 2010; 33:137-150. DOI: 10.1017/s0140525x09991567 [PubMed: 20584369]

Epskamp S, Borsboom D, Fried E. Estimating psychological networks and their stability: A tutorial paper. Behavior Research Methods. 2017; doi: 10.3758/s13428-017-0862-1

Forbes M, Wright A, Markon K, Krueger R. Evidence that psychopathology symptom networks have limited replicability. J Abnorm Psychol. 2017; doi: 10.1037/abn0000276

Fried, E., Cramer, A. Moving forward: Challenges and directions for psychopathological network theory and methodology. Perspectives on Psychological Science. in pressRetrieved from https:// osf.io/bnekp

Fried, E., Eidhof, M., Palic, S., Costantini, G., Huisman-van Dijk, H., Bockting, C., ... Karstoft, K-I. Replicability and generalizability of PTSD networks: A cross-cultural multisite study of PTSD symptoms in four trauma patient samples. under reviewRetrieved from https://osf.io/preprints/ psyarxiv/3zq5u/

Fried E, van Borkulo C, Cramer A, Lynn B, Schoevers R, Borsboom D. Mental disorders as networks of problems: a review of recent insights. Soc Psychiatry Psychiatr Epidemiol. 2016; doi: 10.1007/ s00127-016-1319-z

Guloksuz S, Pries L, van Os J. Application of network methods for understanding mental disorders: pitfalls and promise. Psychol Med. 2017:1-10.

Heeren A, McNally R. An integrative network approach to social anxiety disorder: The complex dynamic interplay among attentional bias for threat, attentional control, and symptoms. Journal of Anxiety Disorders. 2016; 42:95-104. doi: http://dx.doi.org/10.1016/j.janxdis.2016.06.009. [PubMed: 27395806]

Hoorelbeke K, Marchetti I, De Schryver M, Koster E. The interplay between cognitive risk and resilience factors in remitted depression: A network analysis. J Affect Disord. 2016; 195:96-104. DOI: 10.1016/j.jad.2016.02.001 [PubMed: 26878206]

McNally R. Can network analysis transform psychopathology? Behav Res Ther. 2016; doi: 10.1016/ j.brat.2016.06.006

McNally R, Heeren A, Robinaugh D. A Bayesian network analysis of posttraumatic stress disorder symptoms in adults reporting childhood sexual abuse. European Journal of Psychotraumatology. 2017; 8:1341276.doi: 10.1080/20008198.2017.1341276 [PubMed: 29038690]

Mitchell K, Wolf E, Bovin M, Lee L, Green J, Rosen R, ... Marx B. Network models of DSM-5 posttraumatic stress disorder: Implications for ICD-11. J Abnorm Psychol. 2017; 126:355. [PubMed: 28191985]

Rhemtulla M, Fried E, Aggen S, Tuerlinckx F, Kendler K, Borsboom D. Network analysis of substance abuse and dependence symptoms. Drug Alcohol Depend. 2016; 161:230-237. DOI: 10.1016/ j.drugalcdep.2016.02.005 [PubMed: 26898186] 
Robinaugh D, LeBlanc N, Vuletich H, McNally R. Network analysis of persistent complex bereavement disorder in conjugally bereaved adults. J Abnorm Psychol. 2014; 123:510-522. DOI: 10.1037/abn0000002 [PubMed: 24933281]

Spiller T, Schick M, Schnyder U, Bryant R, Nickerson A, Morina N. Symptoms of posttraumatic stress disorder in a clinical sample of refugees: A network analysis. European Journal of Psychotraumatology. 2017; 8:1318032. [PubMed: 29038688]

Steinley D, Hoffman M, Brusco M, Sher K. A method for making inferences in network analysis: Comment on Forbes, Wright, Markon, and Krueger (2017). J Abnorm Psychol. 2017

Sullivan C, Smith A, Lewis M, Jones R. Network analysis of PTSD symptoms following mass violence. Psychol Trauma. 2016

Terluin B, de Boer M, de Vet H. Differences in connection strength between mental symptoms might be explained by differences in variance: Reanalysis of network data did not confirm staging. PLoS One. 2016; 11:e0155205. [PubMed: 27880771]

Wichers M, Wigman J, Bringmann L, de Jonge P. Mental disorders as networks: Some cautionary reflections on a promising approach. Soc Psychiatry Psychiatr Epidemiol. 2017; 52:143-145. [PubMed: 28210750] 


\section{General Scientific Summary}

This response to the two commentaries on our target article reiterates the conclusion that popular network analysis methods produce unreliable results. 


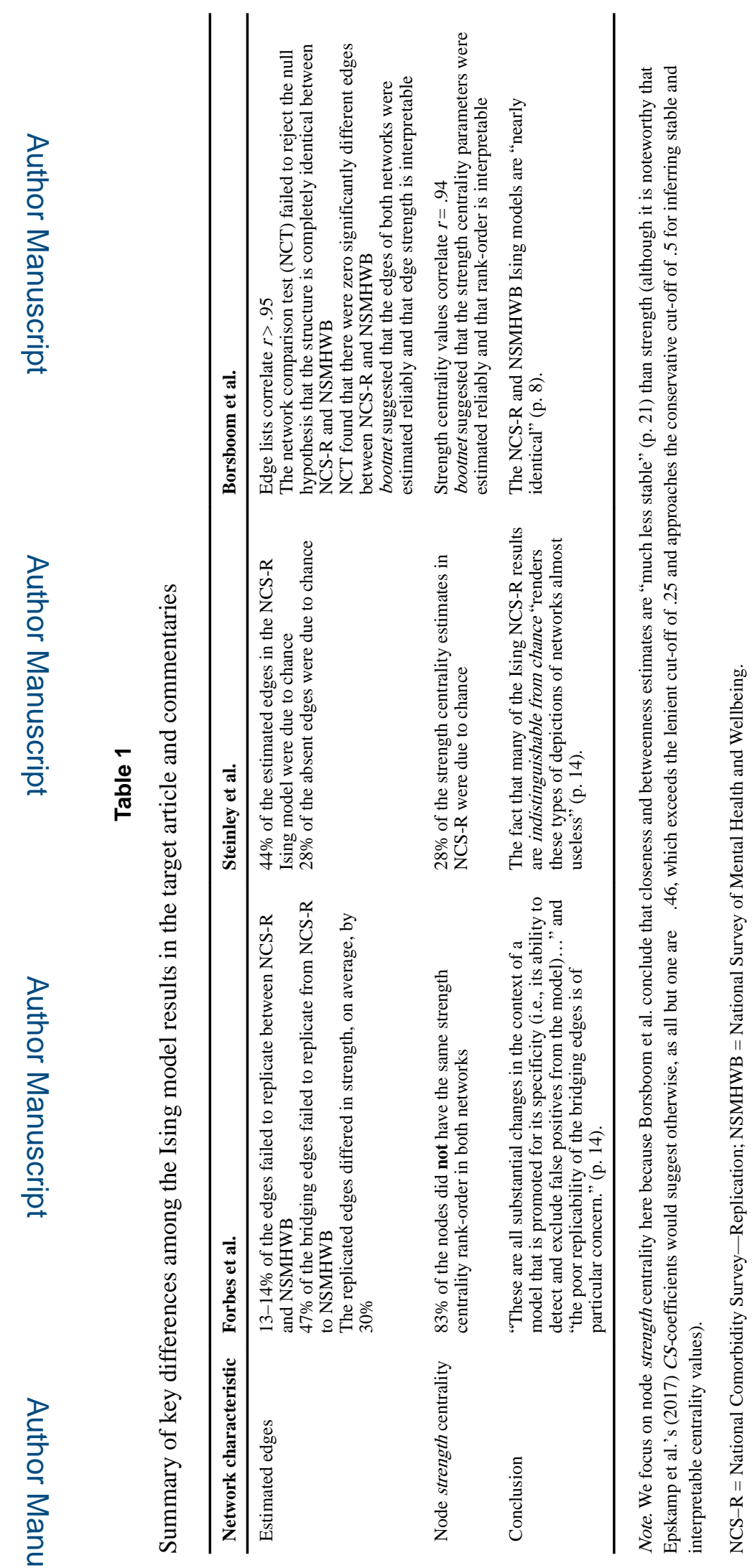




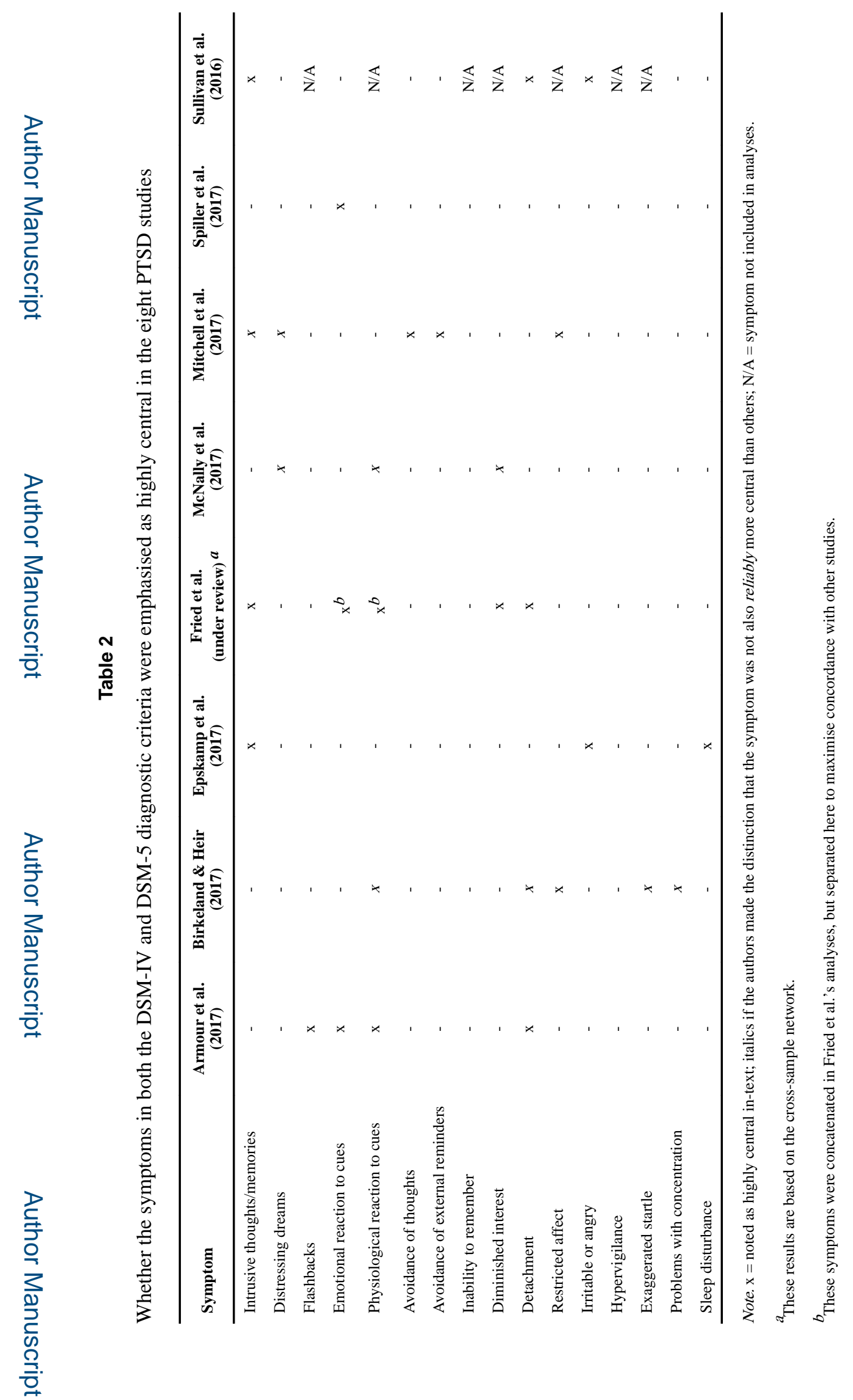

J Abnorm Psychol. Author manuscript; available in PMC 2018 October 01. 
Forbes et al.

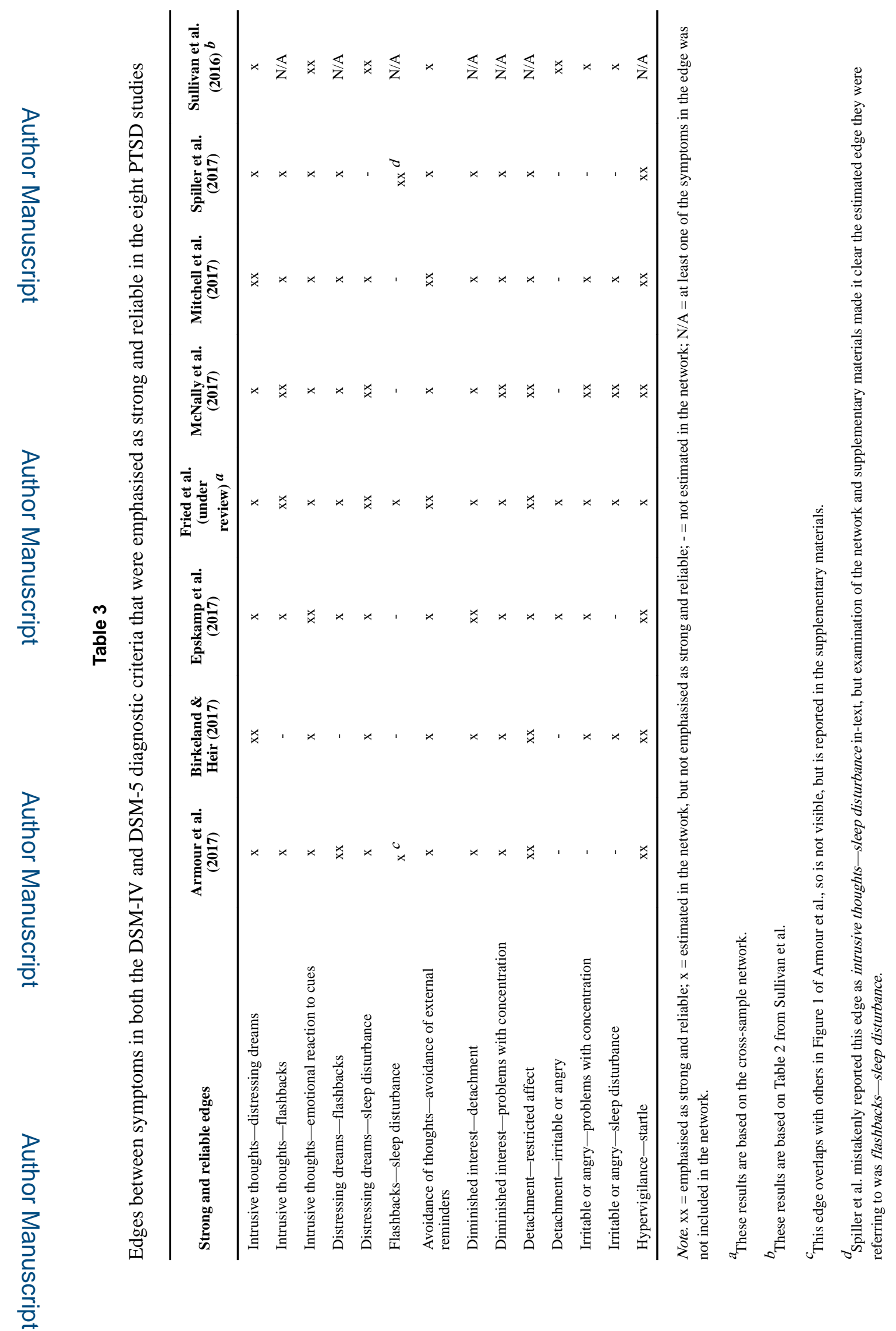

J Abnorm Psychol. Author manuscript; available in PMC 2018 October 01. 